\title{
Hybrid Systems for Power Quality Control in Distribution Networks
}

\author{
Valery P. Dovgun*, Sergey A. Temerbaev, \\ Natalia P. Boyarskaya and Maxim O. Chernyshov \\ Siberian Federal University \\ 79 Svobodny, Krasnoyarsk, 660041, Russia
}

In this paper hybrid power filters, intended for power quality control in distribution systems with nonlinear loads are considered. It is shown that multifunction devices may be realized on the basis of hybrid power filters. Such devices can accomplish voltage and current harmonic mitigation, correction of network frequency responses, compensating of the reactive power in the point of common coupling.

Keywords: power quality, nonlinear load, harmonics, hybrid power filter.

DOI: 10.17516/1999-494X-2015-8-8-997-1009.

\section{Гибридные системы}

\section{управления качеством электроэнергии \\ в распределительных сетях}

\author{
В.П. Довгун, С.А. Темербаев, \\ Н.П. Боярская, М.О. Чернышов \\ Сибирский федеральный университет \\ Россия, 660041, Красноярск, пр. Свободный, 79
}

\begin{abstract}
В статье рассмотрены гибридные фильтрокомпенсирующие устройства, предназначенные для управления качеством электроэнергии в распределительных сетях с высоким уровнем нелинейной нагрузки. Показано, что на основе гибридных силовых фильтров возможно создание многофункииональных устройств, обеспечивающих ослабление высших гармоник токов и напряжений, коррекиию частотных характеристик сети, а также регулирование напряжения и реактивной мощности в точке общего присоединения фильтра и нелинейной нагрузки.
\end{abstract}

Ключевые слова: качество электроэнергии, нелинейная нагрузка, гармоники, гибридные силовые фильтры.

(c) Siberian Federal University. All rights reserved

* Corresponding author E-mail address: Vdovgun@sfu-kras.ru 


\section{Введение}

Широкое применение устройств с нелинейными вольтамперными характеристиками приводит к увеличению уровня высших гармоник токов и напряжений в электрических сетях. Результатом воздействия гармоник на систему электроснабжения выступает увеличение потерь во вращающихся машинах, трансформаторах, линиях электропередачи, ускоренное старение изоляции электрооборудования, ложные срабатывания устройств релейной защиты и автоматики [1].

Основным источником гармонических искажений являются нелинейные нагрузки промышленных предприятий. Однако в последние годы отмечается значительное ухудшение качества электрической энергии в сетях коммерческих и офисных потребителей - торговых комплексов, офисных зданий, учебных заведений. Нелинейной нагрузкой таких потребителей служит офисное оборудование, использующее однофазные источники питания (персональные компьютеры, серверы, принтеры, блоки бесперебойного питания и т.п.), а также энергосберегающие системы освещения. У этой группы потребителей доля нелинейной нагрузки может намного превышать линейную составляющую. Результаты исследований, проведенных в [2-4], показывают, что проблема обеспечения качества электроэнергии весьма актуальна для всех систем электроснабжения, включая городские распределительные сети. У крупных коммерческих и офисных потребителей наблюдаются значительные искажения формы токов. В ряде случаев коэффициент искажения синусоидальной формы кривой тока может достигать $30 \%$ [4]. Поэтому меры, направленные на поддержание качества электроэнергии, должны стать составной частью комплекса мероприятий по энергосбережению и повышению энергоэффективности.

Распространенным средством уменьшения уровня высших гармоник в сетях электроснабжения признаны пассивные фильтры гармоник (ПФГ). Недостаток пассивных фильтров заключается в том, что они статические устройства. Их использование в сетях, где большая часть нелинейных нагрузок имеет переменный характер, оказывается неэффективным. Для управления качеством электроэнергии в распределительных сетях необходимо создание адаптивных устройств, обеспечивающих управление основными показателями, определяющими эффективность и качество электроснабжения.

Такими адаптивными устройствами являются активные силовые фильтры (АФ) $[2,5]$. АФ представляет коммутируемое устройство, характеристики которого формируются с помощью специального закона управления. Однако широкое применение АФ ограничивается их сложностью и высокой стоимостью. Для эффективного ослабления высших гармоник активный фильтр должен иметь значительную мощность, сравнимую с мощностью нелинейной нагрузки. Поэтому использование силовых активных фильтров может оказаться экономически нецелесообразным.

Более перспективны гибридные фильтрокомпенсирующие устройства (ФКУ), представляющие сочетание пассивного и активного фильтров. Гибридные ФКУ обладают основными достоинствами пассивных и активных фильтров. В то же время использование гибридных устройств обеспечивает следующие преимущества.

Значительно уменьшается мощность активного фильтра, и за счет этого снижается стоимость всего устройства. 
По сравнению с пассивными фильтрами повышается эффективность компенсации высших гармоник напряжения и тока при изменении характеристик нелинейной нагрузки.

Одна из серьезных проблем пассивных фильтров - нежелательные резонансные явления, возникающие между ветвями ПФ и индуктивностью сети. Использование активного фильтра позволяет демпфировать характеристики гибридного устройства и ослабить резонансные явления между пассивным фильтром и сетью.

Уменьшается негативное влияние изменения параметров на компенсационные характеристики фильтра.

Важное достоинство гибридных фильтров заключается в том, что их можно использовать в сетях, где уже установлены пассивные ФКУ. В этом случае активный фильтр небольшой мощности используется в качестве средства управления характеристиками пассивных устройств $[5,6]$.

«Чистые» активные фильтры могут выполнять одновременно несколько функций: фильтрацию гармоник, коррекцию частотных характеристик сети, компенсацию реактивной мощности и т.д. Пассивные фильтры используются для компенсации реактивной мощности и подавления высших гармоник, создаваемых нелинейной нагрузкой. В то же время сфера применения гибридных фильтров ограничена преимущественно фильтрацией высших гармоник [2].

Цель статьи - исследование возможности использования гибридных фильтров гармоник в качестве многофункциональных фильтрокомпенсирующих устройств, осуществляющих подавление высших гармоник, создаваемых нелинейной нагрузкой и внешней сетью, а также регулирование реактивной мощности и напряжения в точке общего присоединения фильтра и нелинейной нагрузки. Проанализировано влияние пассивного и активного фильтров на характеристики гибридного устройства. Предложена стратегия управления характеристиками гибридного фильтра, обеспечивающая ослабление высших гармоник токов и напряжений, коррекцию частотных характеристик сети, а также регулирование реактивной мощности и напряжения в точке общего присоединения.

\section{Топология гибридных фильтрокомпенсирующих устройств}

Существуют различные конфигурации гибридных фильтрокомпенсирующих устройств, отличающихся способом включения активного и пассивного фильтра, а также типом управляющей переменной. Анализ основных конфигураций гибридных фильтров, проведенный в [7], показал, что компенсационные характеристики гибридных ФКУ не являются простой суммой характеристик активной и пассивной частей фильтра. Они зависят как от схемы соединения этих частей, так и от типа управляющей переменной.

Рассмотрим гибридные силовые фильтры, обеспечивающие компенсацию высших гармоник тока сети, а также напряжения в точке общего присоединения ФКУ и нелинейной нагрузки. Учтем, что источником высших гармоник может быть не только нелинейная нагрузка в точке общего присоединения, но и внешняя сеть.

При анализе используем следующие допущения.

Нелинейная нагрузка на частоте $k$-й гармоники имеет характеристики идеального источника тока, обладающего бесконечным внутренним сопротивлением.

$$
-999-
$$


В общем случае пассивный фильтр гармоник может быть включен параллельно нагрузке в точке общего присоединения или последовательно с сопротивлением сети. В статье рассмотрена только параллельная схема включения пассивного фильтра, как наиболее часто встречающаяся на практике.

Активный фильтр моделируется управляемым источником напряжения или тока.

Управляющей величиной активного фильтра является ток нелинейной нагрузки, ток сети или напряжение в точке общего присоединения.

Управляющий параметр АФ имеет частотную характеристику идеального режекторного фильтра, настроенного на частоту сети:

$$
\begin{aligned}
& R(\omega)=R_{\mathrm{aq}}, \quad \omega \neq \omega_{\mathrm{c}} \\
& R(\omega)=0, \quad \omega=\omega_{\mathrm{c}} .
\end{aligned}
$$

Рассмотрим основные конфигурации гибридных силовых фильтров.

Последовательная структура с активным фильтром, управляемым током сети. Эквивалентная схема фильтра на частоте $k$-й гармоники показана на рис. 1 . Здесь $Z_{\text {c }}$ - сопротивление внешней сети на частоте $k$-й гармоники. Управляющий параметр активного фильтра $R_{\text {аф }}$ имеет размерность сопротивления. Нелинейная нагрузка моделируется источником тока $J_{k}$. Источник напряжения $E_{k}$ учитывает гармоническую составляющую, генерируемую внешней сетью. Здесь и далее в качестве тока $I_{\mathrm{c}}$ будем рассматривать $k$-ю гармонику тока сети, а в качестве напряжения $U_{\text {топ }}-k$-ю гармонику напряжения в точке общего присоединения фильтра и нелинейной нагрузки.

Представим систему «фильтр-внешняя сеть» четырехполюсником, на внешних зажимах которого действуют источники гармоник $E_{k}$ и $J_{k}$ (на рис. 1 выделен пунктиром). Для описания четырехполюсника используем уравнения в гибридных параметрах

$$
\left[\begin{array}{c}
I_{\mathrm{c}} \\
U_{\text {топ }}
\end{array}\right]=\left[\begin{array}{ll}
F_{11} & F_{12} \\
F_{21} & F_{22}
\end{array}\right]\left[\begin{array}{c}
E_{k} \\
J_{k}
\end{array}\right] .
$$

Для схемы на рис. 1 матрица гибридных параметров

$$
[F]=\left[\begin{array}{cc}
\frac{1}{Z_{\text {пф }}+Z_{c}+R_{\text {аф }}} & \frac{Z_{\text {пф }}}{Z_{\text {пф }}+Z_{c}+R_{\text {аф }}} \\
\frac{Z_{\text {пф }}+R_{\text {аф }}}{Z_{\text {пा }}+Z_{c}+R_{\text {аф }}} & \frac{Z_{\text {пф }} Z_{\text {с }}}{Z_{\text {пф }}+Z_{c}+R_{\text {аф }}}
\end{array}\right] .
$$

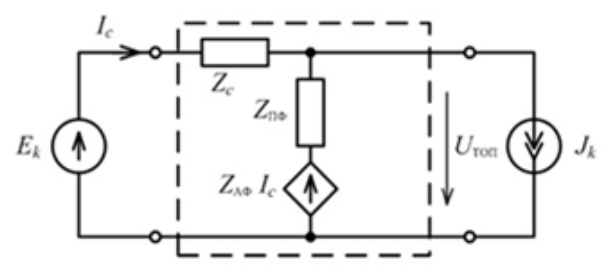

Рис. 1. Последовательная структура с АФ, управляемым током сети

$$
-1000-
$$


Пассивный фильтр представляет собой $L C$-двухполюсник, образованный параллельным соединением резонансных контуров. Операторное входное сопротивление фильтра - дробнорациональная функция с простыми нулями и полюсами, ограниченными осью $ј \omega$. Нули и полюсы входной функции пассивного двухполюсника чередуются. Входная функция $L C$ двухполюсника определяется выражением

$$
Z(s)=H \frac{\prod_{i=1}^{n}\left(s^{2}+\omega_{i}^{2}\right)}{\prod_{j=1}^{n-1}\left(s^{2}+\omega_{j}^{2}\right)}=H \frac{N(s)}{D(s)} .
$$

Здесь $\omega_{i}$ и $\omega_{j}-$ нули и полюсы входной функции. Постоянную $H$ называют коэффициентом нормирования. Если фильтр не содержит одиночных индуктивностей и емкостей, в точках $\mathrm{s}=0$ и $\mathrm{s}=\infty$ расположены полюсы $Z(s)$. Иными словами, полюс $\omega_{1}=0$.

С учетом формулы (3) выражение, определяющее матрицу гибридных параметров структуры на рис. 1, примет вид

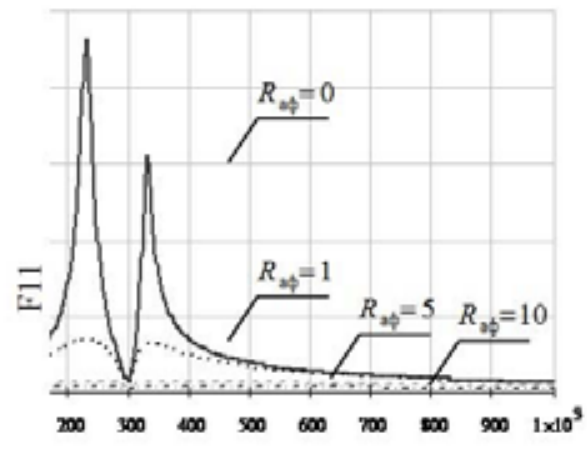

Частота, Гц

$a$

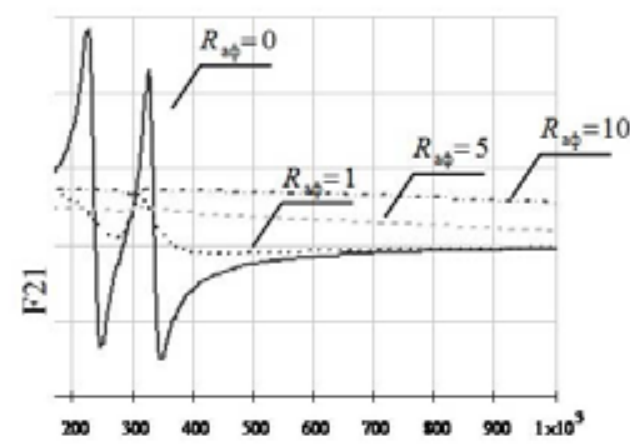

Частота, Гц

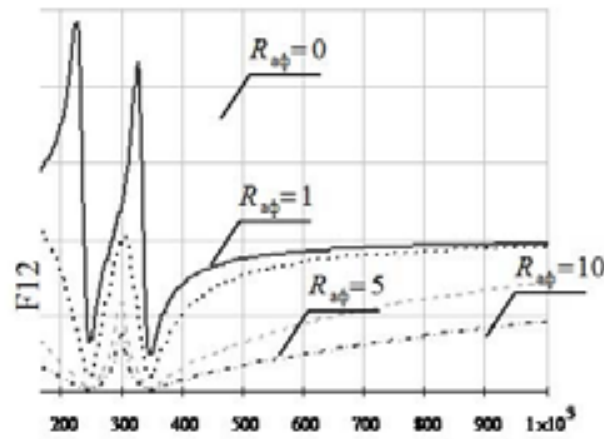

Частота, Гц

6

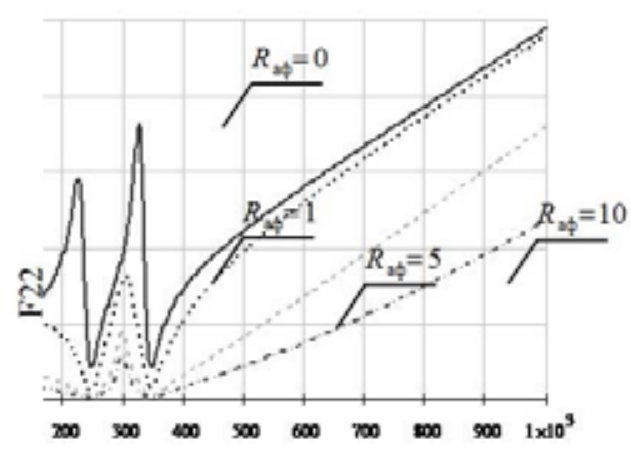

Частота, Гц

6

Рис. 2. Частотные характеристики гибридных параметров последовательной структуры с АФ, управляемым током сети 


$$
[F]=\left[\begin{array}{cc}
\frac{D(s)}{N(s)+D(s)\left(Z_{c}+R_{\mathrm{aq}}\right)} & \frac{N(s)}{N(s)+D(s)\left(Z_{c}+R_{\mathrm{aq}}\right)} \\
\frac{N(s)+R_{\mathrm{aq}} D(s)}{N(s)+D(s)(s)\left(Z_{c}+R_{\mathrm{aq}}\right)} & \frac{N(s) Z_{c}}{N(s)+D(s)\left(Z_{c}+R_{\mathrm{aq}}\right)}
\end{array}\right]
$$

В формуле (4) принято, что коэффициент нормирования $H=1$. Из (4) следует, что элементы матрицы гибридных параметров являются дробно-рациональными функциями комплексной переменной $s$. Их нули расположены на мнимой оси. Частоты нулей параметров $F_{12}$ и $F_{22}$ совпадают с частотами нулей $Z_{\text {пф }}(s)$. Нули параметра $F_{11}$ образованы полюсами $Z_{\text {пф }}(s)$.

В соответствии с (2) и (4) действие активного фильтра на частотах высших гармоник экви-

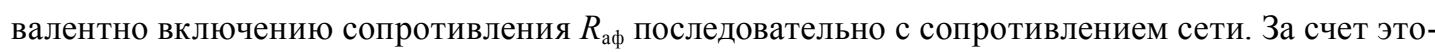
го уменьшается добротность параллельного колебательного контура, образуемого фильтром и индуктивностью сети.

Частотные характеристики коэффициентов матрицы $[F]$ для различных значений параметра активного фильтра показаны на рис. 2. Во всех случаях, рассмотренных в статье, пассивный фильтр образован параллельным соединением двух звеньев, настроенных на частоты пятой и седьмой гармоник. Частота второго полюса $Z_{\text {пф }}(s)$ равна 300 Гц.

Последовательная структура, управляемая током сети, ослабляет высшие гармоники тока, генерируемые как нелинейной нагрузкой, так и внешней сетью. Компенсационные характеристики такой структуры изложены в $[6,8]$. Однако в качестве источника высших гармоник авторами рассматривалась только нелинейная нагрузка в точке общего присоединения. Проведенный анализ показывает, что последовательная структура на рис. 2 не ослабляет высшие гармоники напряжения, распространяющиеся по внешней сети.

Параллельная структура с активным фильтром, управляемым напряжением в точке общего присоединения (рис. 3)

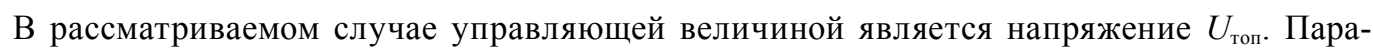
метр активного фильтра $G_{\text {aф }}$ имеет размерность проводимости. Матрица гибридных параметров параллельной структуры

$$
[F]=\left[\begin{array}{cc}
\frac{Y_{\mathrm{c}}\left(Y_{\text {пф }}+G_{\text {аф }}\right)}{Y_{\mathrm{c}}+Y_{\text {пф }}+G_{\text {аф }}} & \frac{Y_{\mathrm{c}}}{Y_{\mathrm{c}}+Y_{\text {пф }}+G_{\text {аф }}} \\
\frac{Y_{\mathrm{c}}}{Y_{\mathrm{c}}+Y_{\text {пा }}+G_{\text {аф }}} & -\frac{1}{Y_{\mathrm{c}}+Y_{\text {пा }}+G_{\text {аф }}}
\end{array}\right] .
$$

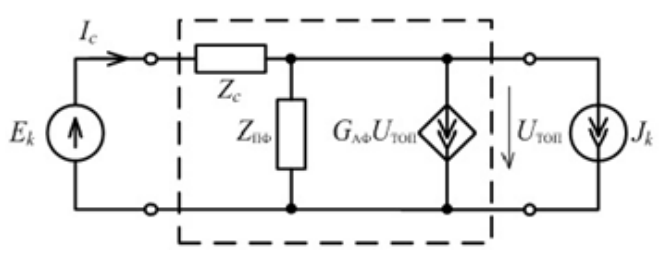

Рис. 3. Параллельная структура с АФ, управляемым напряжением 
Учитывая, что $Y_{\text {пф }}(s)=D(s) / N(s)$, запишем матрицу $[F]$ в виде

$$
[F]=\left[\begin{array}{cc}
\frac{Y_{\mathrm{c}}\left(D(s)+G_{\mathrm{aq}} N(s)\right)}{D(s)+N(s)\left(Y_{\mathrm{c}}+G_{\mathrm{aq}}\right)} & \frac{Y_{\mathrm{c}} N(s)}{D(s)+N(s)\left(Y_{\mathrm{c}}+G_{\mathrm{aq}}\right)} \\
\frac{Y_{\mathrm{c}} N(s)}{D(s)+N(s)\left(Y_{\mathrm{c}}+G_{\mathrm{aq}}\right)} & -\frac{N(s)}{D(s)+N(s)\left(Y_{\mathrm{c}}+G_{\mathrm{aq}}\right)}
\end{array}\right] .
$$

Как и в предыдущем случае, гибридные параметры служат дробно-рациональными функциями переменной $s$. Нули параметров $F_{12}, F_{21}$ и $F_{22}$ формирует пассивный фильтр. На частотах высших гармоник действие активного фильтра эквивалентно включению проводимости $G_{\text {аф }}$ параллельно проводимости сети.

Частотные характеристики гибридных параметров параллельной структуры показаны на рис. 4. В отличие от последовательной схемы, управляемой током, параллельная структура на рис. 3 эффективно ослабляет гармоники напряжения в точке общего присоединения, а также гармоники тока, создаваемые нелинейной нагрузкой. Однако она не может компенсировать гармонические составляющие тока сети, генерируемые внешним источником. Из рис. $4 a$ следует, что при $G_{\text {aф }}>1$ происходит усиление гармоник тока, создаваемых сетью.

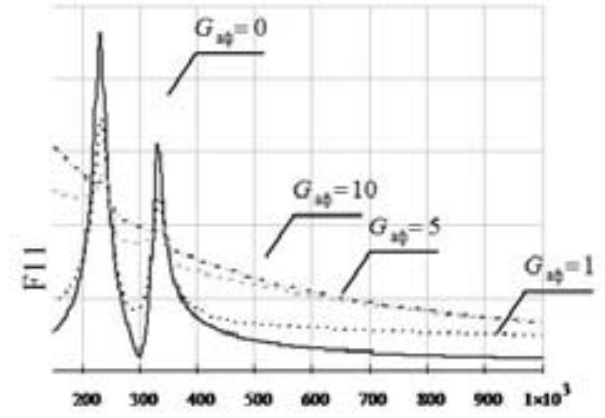

Частота, Ги

$a$

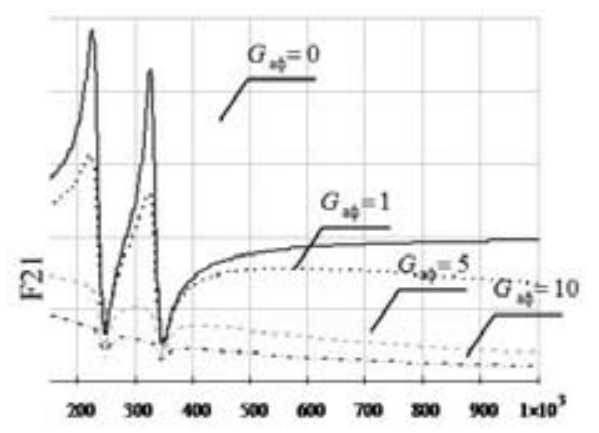

Частота, Ги

$\epsilon$

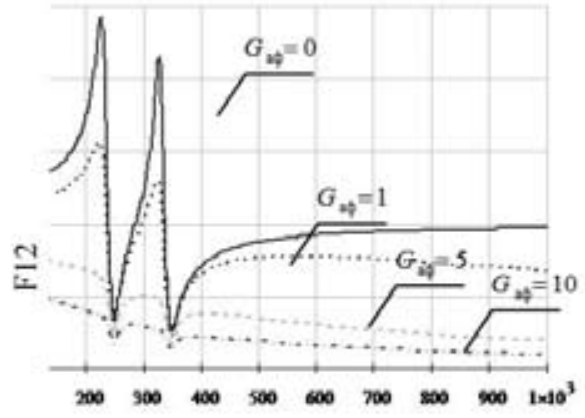

Частота, Ги

6

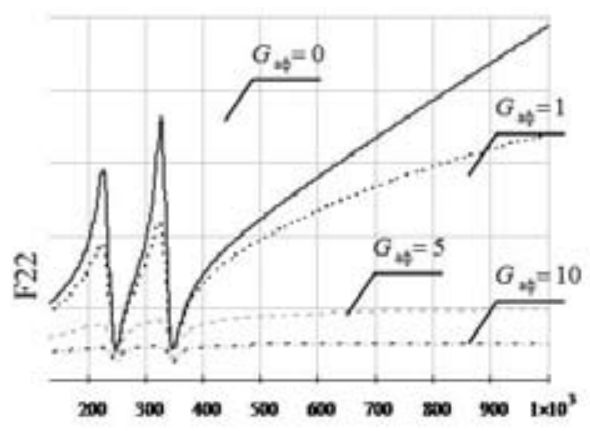

Частота, Ги

2

Рис. 4. Частотные характеристики гибридных параметров параллельной структуры с АФ, управляемым напряжением (рис. 3) 
Комбинированная структура с активным фильтром,

управляемым током сети (рис. 5)

В этом случае пассивный фильтр включен параллельно нагрузке, а активный - последовательно с сопротивлением сети. Силовая часть АФ включается в сеть через согласующий трансформатор. Напряжение активного фильтра пропорционально току сети.

Для схемы на рис. 5 матрица гибридных параметров

$$
[F]=\left[\begin{array}{cc}
\frac{1}{Z_{\text {пф }}+Z_{c}+R_{\text {аф }}} & \frac{Z_{\text {пф }}}{Z_{\text {пф }}+Z_{c}+R_{\text {аф }}} \\
\frac{Z_{\text {пф }}}{Z_{\text {пф }}+Z_{c}+R_{\text {аф }}} & -\frac{Z_{\text {пф }}\left(Z_{c}+R_{\text {аф }}\right)}{Z_{\text {пф }}+Z_{c}+R_{\text {аф }}}
\end{array}\right] .
$$

Учитывая, что $Z_{\text {пф }}=N(s) / D(s)$, запишем матрицу $[F]$ в виде

$$
[F]=\left[\begin{array}{cc}
\frac{D(s)}{N(s)+D(s)\left(Z_{c}+R_{\mathrm{aq}}\right)} & \frac{N(s)}{N(s)+D(s)\left(Z_{c}+R_{\mathrm{aq}}\right)} \\
\frac{N(s)}{N(s)+D(s)\left(Z_{c}+R_{\mathrm{aq}}\right)} & -\frac{N(s)\left(Z_{c}+R_{\mathrm{aq}}\right)}{N(s)+D(s)\left(Z_{c}+R_{\mathrm{aq}}\right)}
\end{array}\right] .
$$

Частотные характеристики гибридных параметров комбинированной структуры представлены на рис. 6.

Из формул (5) и (6) следует, что действие активного фильтра в схеме на рис. 5 эквива-

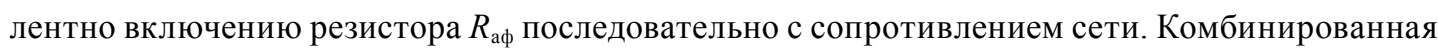
структура с активным фильтром, управляемым током, компенсирует искажения тока сети, вызванные как нелинейной нагрузкой, так и внешней сетью. Однако ослабление гармоник напряжения, создаваемых нелинейной нагрузкой, происходит только на частотах нулей сопротивления $Z_{\text {пф }}$. Поскольку в рассматриваемом примере пассивный фильтр настроен на частоты пятой и седьмой гармоник, будет происходить усиление высокочастотных $(n>7)$ гармоник в спектре напряжения $U_{\text {топ }}$ Это хорошо иллюстрирует частотная характеристика параметра $F_{22}$ (рис. 62).

Проведенный анализ позволяет оценить влияние пассивного и активного фильтров на компенсационные характеристики гибридного устройства. Частоты минимумов и максимумов гибридных параметров рассмотренных структур определяются расположением нулей и полюсов операторного сопротивления пассивного фильтра. Действие активного фильтра на частотах высших гармоник эквивалентно включению активного сопротивления последовательно или параллельно сопротивлению сети. Это уменьшает добротность параллельного

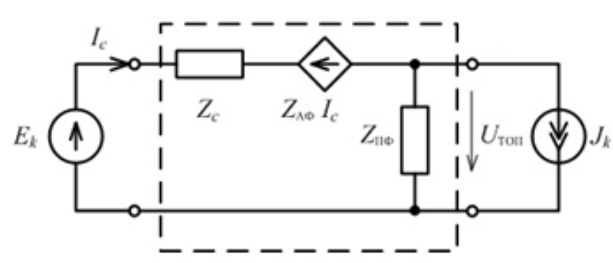

Рис. 5. Комбинированная структура с АФ, управляемым током

$$
-1004-
$$




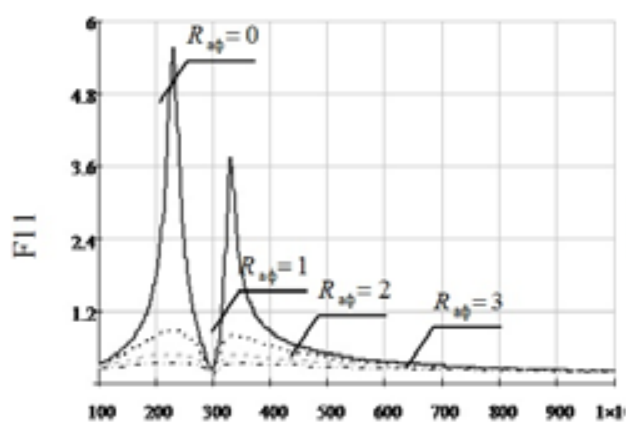

Частота, Гц

$a$

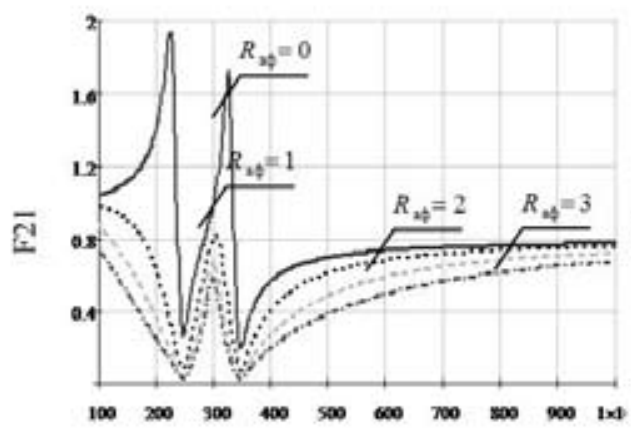

Частота, Ги

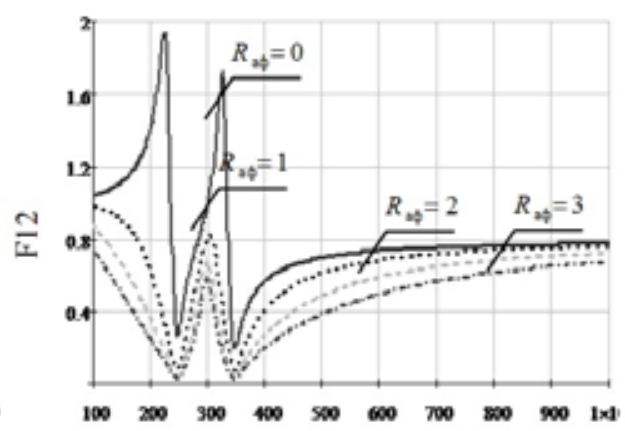

Частота, Гц

6

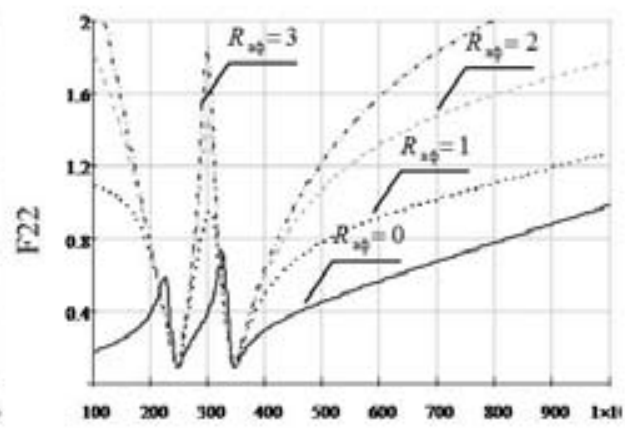

Частота, Ги

Рис. 6. Частотные характеристики гибридных параметров комбинированной структуры (рис. 5)

колебательного контура, образуемого индуктивностью сети и ветвями пассивного фильтра, ослабляет нежелательные резонансные явления между фильтром и сетью.

Отметим, что в некоторых случаях включение активного фильтра ухудшает компенсационные характеристики гибридного устройства. Например, последовательная структура с активным фильтром, управляемым током сети (рис. 1), не ослабляет высшие гармоники напряжения, распространяемые внешней сетью, в отличие от «чистого» пассивного фильтра.

Проведенный анализ позволяет определить область применения перечисленных структур. Все рассмотренные конфигурации гибридных фильтров эффективно ослабляют высшие гармоники тока, создаваемые нелинейной нагрузкой в точке общего присоединения. Однако по отношению к гармоникам тока и напряжения, создаваемым внешней сетью, они ведут себя по-разному.

Последовательная схема с АФ, управляемым током, и комбинированная структура эффективны в тех случаях, когда необходимо ослабить гармоники тока сети, создаваемые как нелинейной нагрузкой, так и внешними источниками.

Параллельную структуру с АФ, управляемым напряжением, целесообразно использовать в тех случаях, когда требуется ослабить распространение высших гармоник напряжения, ге- 
нерируемых нелинейной нагрузкой и внешней сетью. Отметим, что параллельный активный фильтр, управляемый напряжением в точке подключения, впервые предложено использовать для компенсации распространения гармоник напряжения в радиальной сети в работе [9]. Применение гибридной схемы позволяет уменьшить мощность активного фильтра и за счет этого удешевить компенсирующее устройство.

\section{Регулирование реактивной мощности \\ и напряжения в точке подключения}

Помимо несинусоидальности токов и напряжений важным параметром, определяющим качество электроэнергии, являются отклонения и колебания напряжения в узлах сети. Для комплексного решения проблемы необходимы многофункциональные устройства, воздействующие одновременно на несколько параметров. В [10] они названы многофункциональными оптимизирующими устройствами.

Рассмотрим возможность использования гибридных ФКУ для регулирования напряжения и реактивной мощности в точке подключения. Сопротивление пассивного фильтра на частоте основной гармоники имеет емкостный характер, поэтому гибридное ФКУ отдает в сеть реактивную мощность. Величину реактивной мощности можно изменять, регулируя напряжение или ток пассивного фильтра на частоте основной гармоники.

Для одновременного регулирования реактивной мощности и компенсации гармонических составляющих токов и напряжений управляющие сигналы гибридного ФКУ должны включать компоненты, имеющие частоту основной и высших гармоник. Эквивалентные схемы компенсирующих устройств, обеспечивающих такое регулирование, показаны на рис. 7.

На рис. $7 Z_{\text {н }}, Z_{\mathrm{c}}, Z_{\text {пф }}$ - сопротивления нагрузки, сети и пассивного фильтра на частоте основной гармоники, $\beta$ - безразмерный коэффициент. Зависимость $\beta$ от частоты имеет характеристику идеального полосно-пропускающего фильтра:

$$
\begin{aligned}
& \beta(j \omega)=\beta, \quad \omega=\omega_{1}, \\
& \beta(j \omega)=0, \quad \omega \neq \omega_{1} .
\end{aligned}
$$

При выполнении условия $\left|Z_{\mathrm{H}}\left(j \omega_{1}\right)\right|>>\left|Z_{\mathrm{c}}\left(j \omega_{1}\right)\right|$ для обеих конфигураций напряжение в точке общего присоединения на частоте основной гармоники определяется равенством

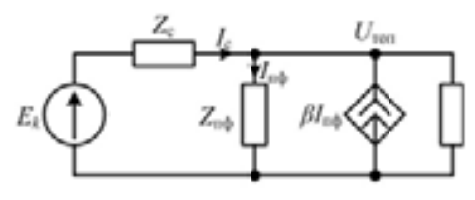

$a$

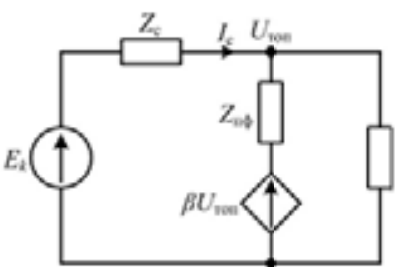

6

Рис. 7. Эквивалентные схемы компенсирующих устройств, регулирующих напряжение и реактивную мощность в точке общего присоединения 


$$
U_{\text {топ }}=\frac{Z_{\text {пф }}}{Z_{c}(1-\beta)+Z_{\text {пф }}} E_{\mathrm{c}} .
$$

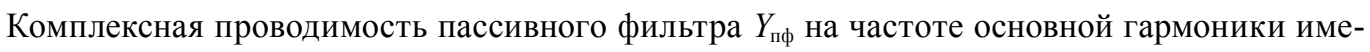
ет емкостный характер. Из (7) следует, что эквивалентная проводимость компенсирующих устройств на рис. $6 a, \sigma$

$$
Y_{\text {фку }}=(1-\beta) Y_{\text {пф }} .
$$

Изменяя проводимость гибридного ФКУ на частоте основной гармоники, мы получаем возможность регулировать реактивную мощность, генерируемую устройством.

Структуры на рис. $7 a, 6$ могут одновременно обеспечивать регулирование проводимости пассивного фильтра на частоте основной гармоники, а также компенсацию высших гармоник. Для этого можно использовать следующие варианты управляющих сигналов:

для схемы на рис. $7 a$ ток активного фильтра

$$
I_{\text {аф }}=\beta I_{\text {пф }}^{(1)}-G_{\text {aф }} U_{\text {топ }}^{(n)},
$$

для схемы на рис. $7 б$ напряжение активного фильтра

$$
U_{\text {aф }}=\beta U_{\text {топ }}^{(1)}+R_{\mathrm{a \phi}} I_{\mathrm{c}}^{(n)} .
$$

В последних выражениях верхний индекс у токов и напряжений соответствует порядковому номеру гармоники.

Таким образом, рассмотренная комбинированная стратегия управления характеристиками гибридных ФКУ позволяет создать многофункциональные устройства, обеспечивающие подавление высших гармоник и регулирование реактивной мощности, генерируемой фильтром на частоте основной гармоники.

\section{Моделирование характеристик}

\section{гибридных фильтрокомпенсирующих устройств}

Модель последовательной структуры ФКУ с активным фильтром, управляемым током сети, построенная в среде MATLAB, показана на рис. 8.

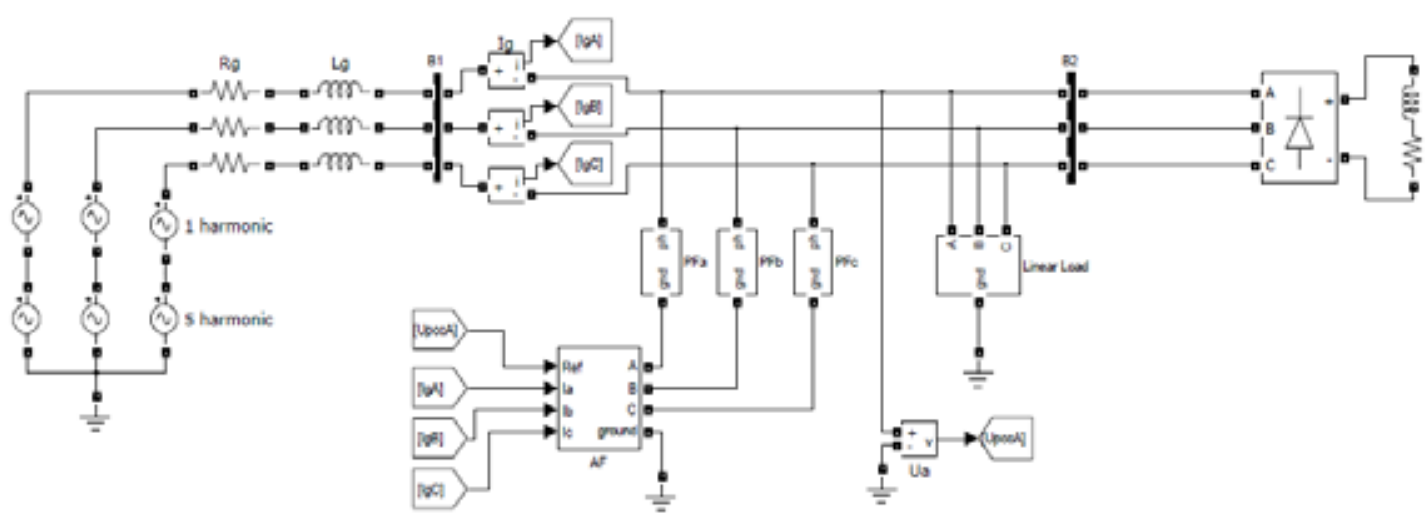

Рис. 8. Модель Simulink трехфазной системы с гибридным фильтром, управляемым током сети 
Поскольку основная задача данной работы - исследование компенсационных возможностей гибридных ФКУ, активный фильтр представлен упрощенной моделью в форме управляемого источника напряжения.

Пассивный фильтр в каждой фазе сети образован параллельным соединением двух резонансных контуров, настроенных на частоты пятой и седьмой гармоник. Активный фильтр $A F$ включен последовательно с пассивным фильтром. Параметр активного фильтра $R_{\text {аф }}=5$ Ом. Внешний источник гармоник, поступающих из сети, моделируется источником синусоидального напряжения частотой 250 Гц, включенным последовательно с основным источником. Блок LinearLoad моделирует линейную нагрузку. Нелинейная нагрузка представлена мостовым выпрямителем с индуктивным сглаживающим фильтром.

Включение пассивного фильтра происходит в момент времени $t=0,2 \mathrm{c}$. Графики тока и напряжения на интервале $0,1-0,55$ с представлены на рис. 9 .

После включения пассивного фильтра происходит увеличение пятой гармоники тока, создаваемой внешним источником. За счет этого коэффициент искажения синусоидальной формы кривой тока сети увеличился с $K_{I}=17,91 \%$ до $K_{I}=22,54 \%$. Коэффициент искажения синусоидальной формы кривой напряжения $K_{U}$ уменьшился до $K_{U}=4,69 \%$.

В момент времени $t=0,4$ с происходит включение активного фильтра.

Активный фильтр компенсирует высшие гармоники тока, вызванные как внешним источником, так и нелинейной нагрузкой, поэтому коэффициент искажения синусоидальной формы кривой тока уменьшается с $K_{I}=22,54 \%$ до $K_{I}=5,26 \%$. Однако коэффициент искажения формы кривой напряжения увеличился до $K_{U}=7,75 \%$.

Моделирование характеристик гибридного фильтра подтвердило результаты анализа, проведенного ранее. Использование последовательного гибридного фильтра, управляемого током сети, целесообразно тех в случаях, когда необходимо компенсировать гармоники тока сети, вызванные как нелинейной нагрузкой, так и внешним источником.

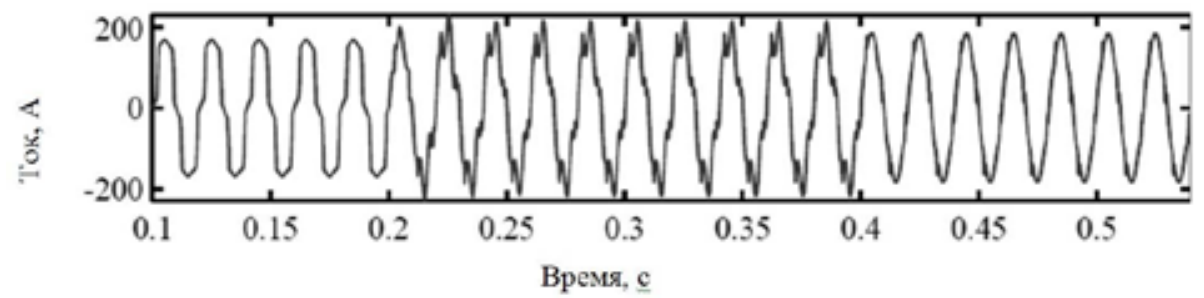

$a$

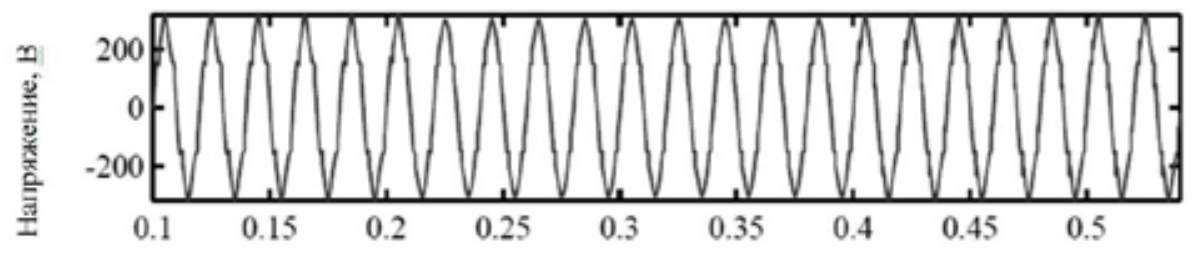

Bpems, c

6

Рис. 9. Ток сети (а) и напряжение в точке общего присоединения (б) 


\section{Выводы}

В статье исследованы возможности использования гибридных фильтров гармоник в качестве многофункциональных фильтрокомпенсирующих устройств, осуществляющих управление несколькими параметрами, определяющими качество электрической энергии в распределительных сетях. Проанализированы компенсационные характеристики основных структур гибридных фильтров при действии гармоник, создаваемых как нелинейной нагрузкой, так и внешней сетью. Показано, что частоты максимумов и минимумов гибридных параметров определяются нулями и полюсами операторного сопротивления пассивного фильтра $Z_{\text {пф }}(s)$.

Предложена стратегия управления характеристиками гибридного фильтра, обеспечивающая подавление высших гармоник напряжения и тока, а также регулирование реактивной мощности на частоте основной гармоники.

Показано, что на основе гибридных силовых фильтров возможно создание многофункциональных устройств, обеспечивающих ослабление гармонических составляющих, а также регулирование реактивной мощности и напряжения в точке общего присоединения.

\section{Список литературы}

[1] Куско А., Томпсон М. Качество энергии в электрических сетях: пер. с англ. М.: ДодэкаXXI, 2008, 336 c. [Kusko A., Thompson M. Power Quality in Electrical Systems, Moscow, DodekaXXI, 2008, 336 p.]

[2] Akagi H. Proceedings of the IEEE, 2005, (93(12), 2128-2141.

[3] De Lima Tostes M., Bezerra U., Silva R. IEEE Transactions on Power Delivery, 2005, 20(1), 384-389.

[4] Темербаев С. А., Боярская Н. П., Довгун В. П. Журнал СФУ. Техника и технологии, 2013, 6(1), 107-120 [Temerbaev S.A., Boiarskaia N.P., Dovgun V.P. J. Sib. Fed. Univ. Eng. technol., 2013 6(1), 107-120]

[5] Розанов Ю.К., Рябчицкий М.В., Кваснюк А.А. Силовая электроника: учебник для вузов. Изд. 2-е. М.: Изд. дом МЭИ, 2009, 632 с. [Rozanov Iu. K., Riabchinskii M.V., Kvasiuk A.A. Power electronics: textbook for high schools. 2-nd ed. Moscow, MPEI, 2009, 632 p.]

[6] Довгун В.П., Темербаев С.А., Егоров Д.Э., Шевченко Е.С. Известия вузов. Проблемь энергетики, 2012, 11-12, 72-80 [Dovgun V.P., Temerbaev S.A., Egorov D.E., Shevchenko E.S. Izvestiya vuzov. The energy problem, 2012, 11-12, 72-80].

[7] Van Zyl A., Enslin J., Spee R. IEEE Transactions on Power Electronics, 1996, 11(5), 691-697.

[8] Rivas D., Moran L., Dixon J., Espinoza J. Improving passive filter compensation performance with active techniques, IEEE Transactions on Power Electronics, 2003, 50(1), 161-169.

[9] Akagi H. IEEE Transactions on Power Delivery, 1997, 12(1), 354-362.

[10] Кудрин Б.И. Электроснабжение. М.: Академия, 2012. 352 с. [Kudrin B.I. Power Delivery. Moscow, Akademiia, 2012. 352 p.]

[11] Бурман А.П., Розанов Ю.К., Шакарян Ю.Г.Управление потоками электроэнергии и повышение эффективности электроэнергетических систем: учебное пособие. М.: Изд. дом МЭИ, 2012. 336 c. [Burman A.P., Rozanov Iu.K., Shakarian Iu.G. Managing the flows of electricity and improving the efficiency of electric power systems: textbook for high schools. Moscow, MPEI, 2012. 336 p.] 